\title{
Design of developable surfaces using the given data points
}

\author{
Kotaro YOSHIDA*, Hidefumi WAKAMATSU*, Eiji MORINAGA**, Seichiro TSUTSUMI*** \\ and Takahiro KUBO ${ }^{\dagger}$ \\ * Graduate School of Engineering, Osaka University \\ Yamadaoka, Suita city, Osaka 565-0871, JAPAN \\ E-mail: kotaro.yoshida@mapse.eng.osaka-u.ac.jp \\ ** Graduate School of Humanities and Sustainable System Sciences, Osaka Prefecture University \\ 1-1, Gakuencho, Naka-ku, Sakai, Osaka 599-8531, Japan \\ *** Joining and Welding Reserach Institute, Osaka University \\ 11-1, Mihogaoka, Ibaraki city, Osaka 567-0047, Japan \\ $\dagger$ Wacoal Holdings Corporation \\ Nakajima-cho, Kisshoin, Minami-ku,Kyoto 601-8530, Japan
}

Received: 1 April 2021; Revised: 14 June 2021; Accepted: 15 July 2021

\begin{abstract}
Many products such as ships, buildings, and clothes contain developable surfaces generated by sweeping a straight line along a three-dimensional curve. An automated design process does not exist due to difficulties satisfying the conditions of a developable surface. This study proposes a method to design a developable surface from the intended data points using the design process of a two-piece brassiere cup as an example. Here, we model a developable surface based on the differential geometry, which converts the design to its boundary curves. Then we formulate the error between the developable surface and the data points from the geometry condition and the optimization problem in the design process. Our method is verified by applying it to three examples: a shape composed of two developable surfaces, a sphere, which is a non-developable surface, and a realistic breast shape.
\end{abstract}

Keywords : Developable surface, Design, Shape optimization, Differential geometry, Automation, CAD

\section{Introduction}

Developable surfaces are used in many industries from shipbuilding to clothes manufacturing because they can be unfolded into a plane without expanding or contracting. Additionally, they can represent surfaces made of leather, paper, or sheet metal. Both the three-dimensional (3D) shape and developed shape must be considered when designing a developable surface. However, designers have difficulty realizing their intentions because satisfying the conditions of a developable surface is challenging. Currently, the intended design is realized based on experience and intuition, which leads to inefficiencies. Many studies have been conducted to address this shortcoming. Previously proposed methods to model developable surfaces have focused on its geodesic line(Bo and Wang, 2007), B-spline or NURBS surface(Aumann, 2003; Pottmann, 1999), offsets of Bertrand curves, which coincide with each normal direction(Ravani and $\mathrm{Ku}, 1991$; Bodduluri and Ravani, 1993), and its arbitrary curves(K.Rose et al.,2007). All of these studies focused on the modeling method and not the design of developable surfaces. If a developable surface can be designed from the 3D shape without considering the condition of developability, the design process will become more efficient. In studies to approximate developable surfaces, Peternell proposed a method to reconstruct a developable surface from its point clouds based on the Laguerre geometry(Peternell, 2004). Chen et al. proposed an algorithm to approximate a developable surface from its point cloud(Chen et al., 1999). Liu et al. proposed a method to approximate surface with meshes composed of planar quadrilaterals(Liu et al., 2006). Stein et al. proposed a method to define the developability of triangle meshes and to approximate them by a set of developable surfaces(Stein et al., 2018), Kilian et al. proposed a method to reconstruct surfaces by curved formation(Kilian et al., 2008) and Mitani et al. proposed a method to design three-dimensional shape from rotational sweep shape(Mitani et al., 2009). These studies investigated approximating surfaces by developable 
surfaces. In the design process, not only must the intended shape be approximated but the condition of the developable surface itself must be satisfied because an inextensible 2D sheet must form the required 3D shape only by bending.

This study focuses on a brassiere cup because the shape is critical. Brassieres are manufactured to meet various demands such as enhancing the breast size, creating cleavage, or minimizing breast movement. Due to these diverse demands, cup shape is a critical element in brassiere design. A brassiere cup is formed by several pieces of cloth, which are called patterns, and a wire. Figure 1 shows an example of a two-piece brassiere cup composed of an upper pattern, a lower pattern, and a lower line.

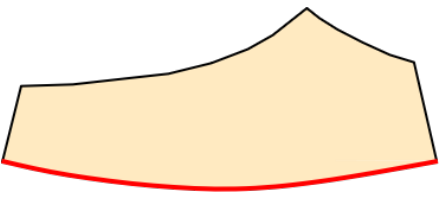

(a) Upper pattern

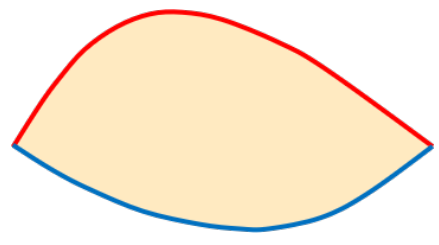

(b) Lower pattern

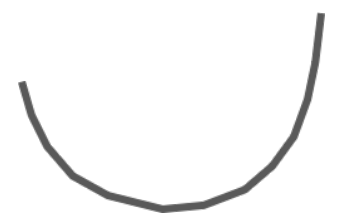

(c) Lower line

Fig. 1 Parts of a two-piece brassiere cup. When designing or modifying the shape of a brassiere cup, the lower and upper patterns must be modified. In the typical design process, modifications are realized according to the designers' experience and intuition(Yoshida et al., 2019).

The three resulting curves are important to the design (Fig.2). The wire line corresponds to the boundary between the breast and body. The ridge line of the cup corresponds to the outline of a bust on a transverse plane, while the upper line connects the cup to the shoulder strap.

In general brassiere makers, a 2D-CAD system is used for pattern design, but the three-dimensional shape of a cup is not designed using a 3D-CAD system. Design of the cup shape and its development to the pattern shape is done in the designers' imagination. Initially, designers consider the intended shape of a brassiere cup. Then they determine the pattern shape. The surface of a worn brassiere cup is not developable. However, the pattern shapes are determined by making a cup made of paper and checking its 3D shape. As paper can be assumed to be inextensible, a paper cup model is composed of several developable surfaces. The physical cup model is used to verify whether the designed pattern shapes form the intended cup shape, including the ridge line. However, pattern shape modification depends on the designers' experience and intuition. Consequently, the paper cup model must be made repeatedly, which leads to inefficiencies.

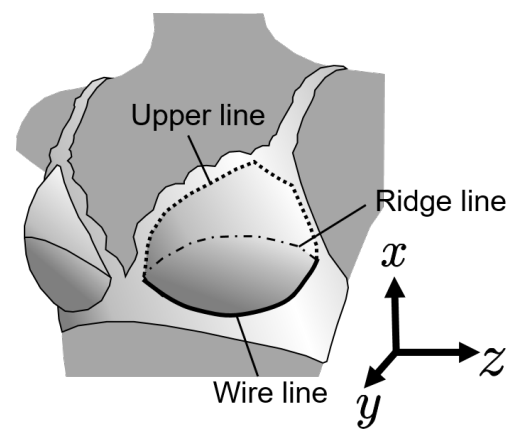

Fig. 2 Expression of a wire, ridge, and upper lines. One is the boundary curve between the breast and body. One outlines the bust on a transverse plane, and the other connects the cup to the shoulder strap. These are important to design the cup shape.

In a study on brassieres, Wakamatsu et al. proposed a method to predict the 3D shape of a paper cup model when the 2D shape of a pattern is given(Wakamatsu et al.,2016,2017). Although patterns can be evaluated without actually creating a paper model, repetitive pattern modifications is still necessary to obtain the target 3D shape of the cup. Ito et al. 
developed a paper model CAD system based on the theory of developable surfaces(Ito et al.,2007). If a 3D curve, which means a sewn curve, and a 2D curve, which means a piece to be sewn, are given, their system can identify the feasible region where the sewn surface does not intersect itself. However, pattern shape design remains a challenge.

We previously proposed a method to design the pattern shape and its developable surface from two lines: a wire line and a ridge line (Yoshida et al., 2019) and the a ridge line design to satisfy its design parameters (Yoshida et al., 2020). These studies focus on the design of a developable surface, but do not evaluate how a surface approximates the given shape. This may lead to inefficiencies. Brassiere patterns are typically comprised of two or three pieces. Herein we focus on a two-piece brassiere cup and propose a method to improve the design efficiency when a cloud of its data points is given.

\section{Modeling of Developable Surface and Its Developed Shape}

This section explains the numerical expressions of a developable surface and its developed shape. In this study, they are formulated based on our proposed method. Generally, a developable surface is a kind of a ruled surface, and the global coordinates of a point on surface $\boldsymbol{X}(s, t)$ can be described by a parametric representation of the form

$$
\boldsymbol{X}(s, t)=\boldsymbol{x}(s)+\operatorname{tg}(s)
$$

where $\boldsymbol{x}$ means a space curve that lies on the surface and $\boldsymbol{g}$ means the direction vector of a generatrix. Therefore, we explain the modeling method of a space curve. Then we discuss the condition to be a developable surface. Figure 3 depicts the normal orthogonal base of a space curve, where $s$ is its arc length and $\zeta$-axis always coincides with its tangential direction. Analogous to the angular velocities of a rigid body(Harashima, 1985), the infinitesimal displacement vector of each axial direction can be described as

$$
\left[\begin{array}{lll}
\boldsymbol{\xi}^{\prime} & \boldsymbol{\eta}^{\prime} & \zeta^{\prime}
\end{array}\right]=\left[\begin{array}{lll}
\boldsymbol{\xi} & \boldsymbol{\eta} & \boldsymbol{\zeta}
\end{array}\right]\left[\begin{array}{ccc}
0 & -\omega_{\zeta} & \omega_{\eta} \\
\omega_{\zeta} & 0 & -\omega_{\xi} \\
-\omega_{\eta} & \omega_{\xi} & 0
\end{array}\right]
$$

where a prime represents a derivative of $s$. Then the position of a space curve $\boldsymbol{x}(s)$ is described as

$$
\boldsymbol{x}(s)=\boldsymbol{x}_{0}+\int_{0}^{s} \zeta d s
$$

Therefore, its deformation can be determined by $\omega=\left[\begin{array}{lll}\omega_{\xi} & \omega_{\eta} & \omega_{\zeta}\end{array}\right]^{\mathrm{T}}$.

The definition of a developable surface is one whose Gaussian curvature $K$ is equal to 0 . The Gaussian curvature is described using its principal curvature $\kappa_{\min }$ and $\kappa_{\max }$ or the fundamental coefficients $E, F, G, L, M$ and $N$ as follows(Kobayashi,1995).

$$
K=\kappa_{\max } \kappa_{\min }=\frac{L N-M^{2}}{E G-F^{2}} .
$$

Here, the fundmental coefficients $E, F, G, L, M$ and $N$ are described by the surface $S(u, v)$ as

$$
\begin{aligned}
& E=\frac{\partial \boldsymbol{S}}{\partial u} \cdot \frac{\partial \boldsymbol{S}}{\partial u}, F=\frac{\partial \boldsymbol{S}}{\partial u} \cdot \frac{\partial \boldsymbol{S}}{\partial v}, G=\frac{\partial \boldsymbol{S}}{\partial v} \cdot \frac{\partial \boldsymbol{S}}{\partial v} \\
& L=\frac{\partial^{2} \boldsymbol{S}}{\partial u^{2}} \cdot \boldsymbol{n}, M=\frac{\partial^{2} \boldsymbol{S}}{\partial u \partial v} \cdot \boldsymbol{n}, N=\frac{\partial^{2} \boldsymbol{S}}{\partial v^{2}} \cdot \boldsymbol{n},
\end{aligned}
$$

where $\boldsymbol{n}$ means a unit normal vector of the surface and is defined as

$$
\boldsymbol{n}=\frac{\frac{\partial \boldsymbol{S}}{\partial u} \times \frac{\partial \boldsymbol{S}}{\partial v}}{\left|\frac{\partial \boldsymbol{S}}{\partial u} \times \frac{\partial \boldsymbol{S}}{\partial v}\right|}
$$

Since generatrices always lie on the surface, its direction is described when the $\eta$-axis always coincides to that of $\boldsymbol{n}$

$$
\boldsymbol{g}=-\zeta \sin \alpha+\xi \cos \alpha
$$

where $\alpha$ describes the angle between a generatrix and $\xi$-axis. From Eqs.(1), (2) and (5), solving $K=0$ gives

$$
\tan \alpha=-\frac{\omega_{\zeta}}{\omega_{\xi}}
$$


Let $\kappa_{\max }$ and $\boldsymbol{d}_{\max }$ be the maximum principal curvature and its direction, and $\kappa_{\min }$ and $\boldsymbol{d}_{\min }$ be the minimum principal curvature and its direction, respectively. Note that $\boldsymbol{d}_{\min }$ always coincides to a generatrix based on the assumption of $\left|\kappa_{\max }\right|>\left|\kappa_{\min }\right|$. The direction of a generatrix is determined by the space curve on a developable surface. Let $s_{L}$ and $s_{U}$ be the arc lengths, $\boldsymbol{x}_{L}\left(s_{L}\right)$ and $\boldsymbol{x}_{U}\left(s_{U}\right)$ be the position vectors, and $\zeta_{L}\left(s_{L}\right)$ and $\zeta_{U}\left(s_{U}\right)$ be the tangential vectors of individual curves. If a line connecting point $\boldsymbol{x}_{L}\left(s_{L}\right)$ and point $\boldsymbol{x}_{U}\left(s_{U}\right)$ coincides with a generatrix, the following equation holds because a generatrix and two tangential vectors of curves at both end points of the generatrix are in one plane(Fig.4)

$$
\operatorname{det}\left(\zeta_{L}, \zeta_{U}, x_{U}-x_{L}\right)=0
$$

Equation (10) is the case of a developable surface, and the arc length of one curve is subordinated to that of the other (i.e. $s_{U}=s_{U}\left(s_{L}\right)$ ). Then angle $\alpha_{L}$ between a generatrix and $\xi_{L}$-axis is calculated following Eq. (8) as

$$
\alpha_{L}=-\sin ^{-1} \boldsymbol{g} \cdot \boldsymbol{\zeta}_{L} \text {. }
$$

Note that $\boldsymbol{g}=\frac{\boldsymbol{x}_{U}-\boldsymbol{x}_{L}}{\left|\boldsymbol{x}_{U}-\boldsymbol{x}_{L}\right|}$. Then, $\omega_{L}$ and $\alpha$ are calculated as

$$
\omega_{L}=\left[\begin{array}{c}
\omega_{\xi_{L}} \\
\omega_{\eta_{L}} \\
\omega_{\zeta_{L}}
\end{array}\right]=\frac{1}{\cos \alpha_{L}}\left[\begin{array}{c}
-\operatorname{det}\left(\boldsymbol{\zeta}_{L}^{\prime}, \boldsymbol{\zeta}_{L}, \boldsymbol{g}\right) \\
\boldsymbol{\zeta}_{L}^{\prime} \cdot \boldsymbol{g} \\
\operatorname{det}\left(\boldsymbol{\zeta}_{L}^{\prime}, \boldsymbol{\zeta}_{L}, \boldsymbol{g}\right) \tan \alpha_{L}
\end{array}\right]
$$

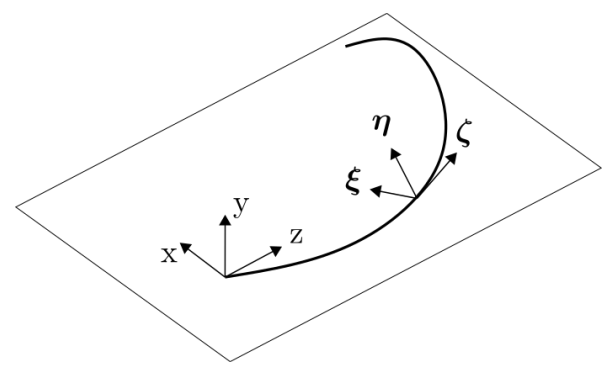

Fig. 3 Object coordinate system on a space curve. Normal orthogonal base whose $\zeta$-axis always coincides with its tangent vector.

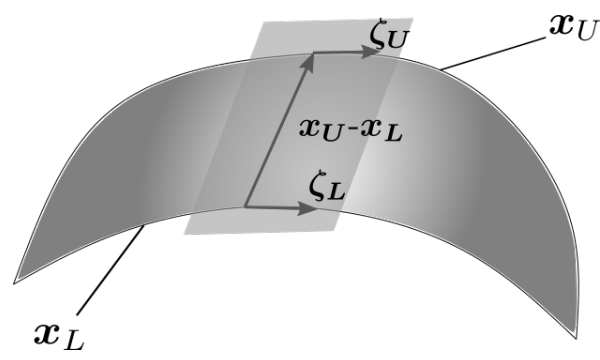

Fig. 4 Condition of a developable surface against its boundary curves. These have the same tangent plane.

Next, we explain a developable surface. $\omega_{\eta_{L}}$ is equal to the geodesic curvature from the definition of the $\eta$-axis. Since deforming a space does not change the geodesic curvature, it corresponds to the planar curvature obtained by developing a space curve. Consider to develop the surface into a $v w$-plane, which is the arbitrary plane in $\mathbb{R}^{3}$. Angle $\mu_{L}$ between the tangential vector of the planar curve corresponding to developed $\partial S_{L}$ and $v$-axis is calculated as

$$
\mu_{L}=\mu_{0}+\int_{0}^{s_{L}} \omega_{\eta_{L}} d s
$$

where $\mu_{0}$ is the initial angle of $\mu$. Then planar position $v_{L}=\left[v_{L}, w_{L}\right]$ is described as

$$
\boldsymbol{v}_{L}=\int_{0}^{s_{L}}\left[\begin{array}{c}
\cos \mu_{L} \\
\sin \mu_{L}
\end{array}\right] d s
$$

and planar position $\boldsymbol{v}_{U}=\left[v_{U}, w_{U}\right]$ of developed $\partial S_{U}$ is calculated as

$$
\boldsymbol{v}_{U}=\boldsymbol{x}_{L}+\left|\boldsymbol{x}_{U}\left(s_{U}\left(s_{L}\right)\right)-\boldsymbol{x}_{L}\left(s_{L}\right)\right|\left[\begin{array}{c}
\cos \left(\mu_{L}+\pi / 2+\alpha_{L}\right) \\
\sin \left(\mu_{L}+\pi / 2+\alpha_{L}\right)
\end{array}\right] \text {. }
$$

From above, a developable surface and its developed surface can be determined by its boundary curves. 


\section{Expression of the design process as an optimization problem}

Here, we explain how our proposed method formulates the error between a developable surface and the given point cloud. Additionally, we convert the design process of a two-piece brassiere cup into an optimization problem. To formulate the error between a developable surface and the given point cloud, the error between a point and the surface is calculated. Error $\varepsilon(\boldsymbol{p})$ between point $\boldsymbol{p}$ and a developable surface expressed by Eq. (1) is defined as

$$
\varepsilon(\boldsymbol{p})=\min _{s, t}|\boldsymbol{p}-\boldsymbol{X}(s, t)|^{2}
$$

When a set of surface parameters $\left(s^{*}, t^{*}\right)$ minimizes Eq. (16), $\boldsymbol{p}-\boldsymbol{X}\left(s^{*}, t^{*}\right)$ is parallel to $\boldsymbol{\eta}$. Therefore, the following equations are satisfied:

$$
\begin{aligned}
& \boldsymbol{d}_{\min } \cdot\left(\boldsymbol{p}-\boldsymbol{X}\left(s^{*}, t^{*}\right)\right)=0 \\
& \boldsymbol{d}_{\max } \cdot\left(\boldsymbol{p}-\boldsymbol{x}\left(s^{*}, t^{*}\right)\right)=0 .
\end{aligned}
$$

By solving Eq. (17), $t^{*}$ is described as

$$
t^{*}=\boldsymbol{d}_{\min } \cdot\left(\boldsymbol{p}-\boldsymbol{X}\left(s^{*}\right)\right)
$$

Using Eq. (19) and solving Eq. (18), $s^{*}$ can be determined. In case of a two-piece brassiere cup, a set of point clouds $D$ must be divided into two sets: $D_{L}$ and $D_{U}$. The former is evaluated by the surface of the lower cup: $S_{L}$, while the latter is evaluated by the surface of the upper cup: $S_{U}$. Note that $D_{U}=D \backslash D_{L}$. To classify point $p_{k}$, let $s_{w}, s_{r}, s_{u}$ be the arc lengths of the lower wire, ridge line, and upper line and let $\omega_{W}\left(s_{w}\right), \omega_{R}\left(s_{r}\right), \omega_{U}\left(s_{u}\right)$ be the vectors to characterize each one, respectively. From Eq. (10), $s_{r}, s_{u}$ can be expressed as functions of $s_{w}: s_{r}\left(s_{w}\right), s_{u}\left(s_{w}\right)$.

Using Eqs. (17) to (19), the nearest surface parameters $\left(s_{w}^{*}, t_{w}^{*}\right)$ of the lower cup to point $\boldsymbol{p}_{k}$ can be derived. Then, nearest point $\boldsymbol{X}\left(s^{*}, t^{*}\right)$ on the lower cup surface to point $\boldsymbol{p}_{k}$ can be obtained. Let $t_{\max }=\left|\boldsymbol{x}_{R}\left(s_{r}\left(s_{w}^{*}\right)\right)-\boldsymbol{x}_{W}\left(s_{w}^{*}\right)\right|$ be the length of a generatrix from the wire line. If $t_{\max }>t_{w}^{*}\left(s_{w}^{*}\right)$,which indicates a projection point perpendicular to point $\boldsymbol{p}_{k}$ to the surface $S_{L}$ is included in the lower cup, point $\boldsymbol{p}_{k}$ is classified to set $D_{L}$. Otherwise, it is classified to set $D_{U}$.

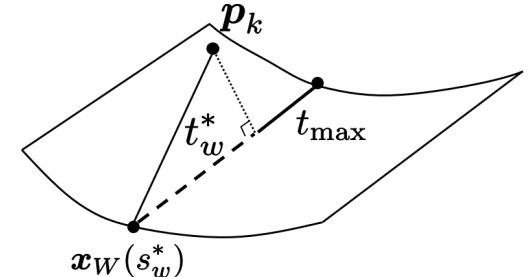

(a) $t^{*} \leq t_{\max }$

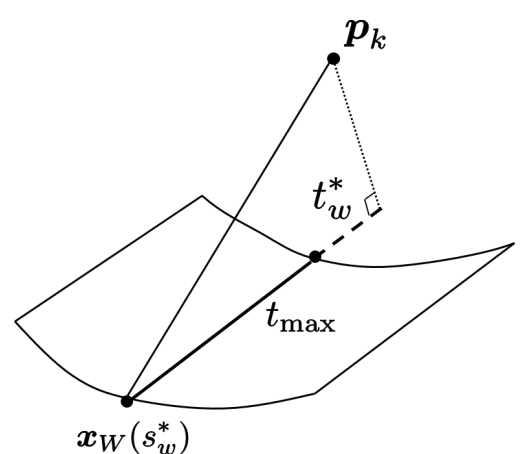

(b) $t^{*}>t_{\max }$

Fig. 5 Relationship between $t^{*}$ and $t_{\max }$. Comparing $t^{*}$ to $t_{\max }$ determines whether $\boldsymbol{p}_{k}$ is evaluated by a lower cup or an upper cup.

The data points are classified into two sets once two developable surfaces for the lower and upper cups are determined. Consequently, the error between the surface of a two-piece brassiere cup and its data points can be formulated as

$$
\Lambda(D)=\sum_{i=1}^{N_{L}} \varepsilon\left(\boldsymbol{p}_{L, i}\right)+\sum_{i=1}^{N_{U}} \varepsilon\left(\boldsymbol{p}_{U, i}\right) .
$$

By finding two developable surfaces that minimize the error in Eq. (20), the appropriate pattern shapes can be determined. In the design process of a brassiere cup, because the wire shape rarely changes, the objective of this optimization problem is to find ridge and upper lines that minimize Eq. (20).

To consider the optimization conditions, we first explain the conditions for the parameters of the ridge and upper lines. $\omega_{\zeta}$ does not affect the shape of the space curve. Therefore, the following conditions are added

$$
\omega_{\zeta_{R}}\left(s_{r}\left(s_{w}\right)\right)=0, \omega_{\zeta_{U}}\left(s_{u}\left(s_{w}\right)\right)=0 \forall s_{w} \in\left[0, L_{L}\right]
$$


With respect to the arc length of each line, the following conditions must be satisfied:

$$
\begin{aligned}
& s_{r}\left(s_{w}\right) \geq 0, s_{u}\left(s_{w}\right) \geq 0 \quad \forall s_{w} \in\left[0, L_{L}\right], \\
& s_{r}^{\prime}\left(s_{w}\right) \geq 0, s_{u}^{\prime}\left(s_{w}\right) \geq 0 \quad \forall s_{w} \in\left[0, L_{L}\right] .
\end{aligned}
$$

In general, the end positions of the ridge and upper lines are aligned to the end point of the wire line. Therefore, when $s_{w}=0, L_{L}$, the following equations must be satisfied

$$
\begin{aligned}
& \boldsymbol{x}_{R}\left(s_{r}\left(s_{w}\right)\right)=\boldsymbol{x}_{L}\left(s_{w}\right), \\
& \boldsymbol{x}_{U}\left(s_{u}\left(s_{w}\right)\right)=\boldsymbol{x}_{L}\left(s_{w}\right) .
\end{aligned}
$$

To guarantee that $S_{L}$ and $S_{U}$ are developable surfaces, the following equations must be satisfied

$$
\begin{aligned}
& \int_{0}^{L_{L}} \operatorname{det}\left(\zeta_{R}\left(s_{r}\left(s_{w}\right)\right), \boldsymbol{\zeta}_{W}\left(s_{w}\right), \boldsymbol{g}\right) d s_{w}=0 \\
& \int_{0}^{L_{L}} \operatorname{det}\left(\boldsymbol{\zeta}_{U}\left(s_{u}\left(s_{w}\right)\right), \boldsymbol{\zeta}_{W}\left(s_{w}\right), \boldsymbol{g}\right) d s_{w}=0 .
\end{aligned}
$$

Next, consider a "fuzzy" condition for a brassiere cup. The upper line has a point connected with the shoulder strap (Fig.6). Hereafter, this point is called the connection point. Typically, the connection point should be as close as possible

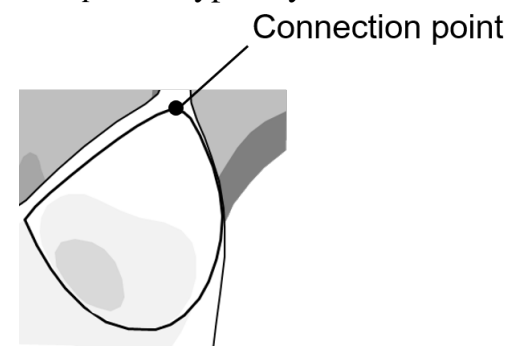

Fig. 6 Expression about the connection point of a brassiere cup given as a fuzzy condition of the cup shape.

to the given target point, but it does not have to match the target point until other constraints such as Eq. (24), which must be satisfied more reliably, can be ignored. Therefore, we propose the following equation to deal with this fuzzy condition $y \simeq Y$

$$
C(y, Y)=k_{1} \exp \left(k_{2}(y-Y)^{2}\right)
$$

where $y$ is a variable, $Y$ is the target value, $k_{1}$ and $k_{2}$ are parameters to adjust the range that satisfies this condition. By taking into account the ambiguity of the constraints in the design process, the feasible region of the design variables can be expanded and the optimization problem can be expected to converge on a better solution. The fuzzy condition can be handled by adding this equation to the objective function. Here, we assume that position $\boldsymbol{X}_{C} \in \mathbb{R}^{3}$, which connects the upper line to the shoulder strap, is given. Then the objective function $V$ is described as

$$
V=\Lambda(\boldsymbol{D})+\sum_{i=0}^{2} C\left(\boldsymbol{x}_{U} \cdot \boldsymbol{e}_{i}, \boldsymbol{X}_{C} \cdot \boldsymbol{e}_{i}\right)
$$

where $\boldsymbol{e}_{0}, \boldsymbol{e}_{1}$, and $\boldsymbol{e}_{2}$ are represented as unit vectors of the $x, y$, and $z$-axis. Solving this optimization under these constraints provides the shape of the brassiere cup from the given data points.

Now we explain how to eliminate the condition and solve an optimization problem. To satisfy Eqs. (22) and (23), $s_{r}\left(s_{w}\right), s_{u}\left(s_{w}\right)$ are described using functions $v_{r}\left(s_{w}\right), v_{u}\left(s_{w}\right)$ as

$$
\begin{aligned}
& s_{r}\left(s_{w}\right)=s_{r_{0}}+\int_{0}^{s_{w}} \exp \left(v_{r}\right) d s_{w}, \\
& s_{u}\left(s_{w}\right)=s_{u_{0}}+\int_{0}^{s_{w}} \exp \left(v_{u}\right) d s_{w} .
\end{aligned}
$$

The initial positions of the ridge and upper lines are assumed to coincide with that of the wire line (i.e., $s_{r_{0}}=s_{u_{0}}=0$ ). Let the composite function of $s_{r}\left(s_{w}\right)$ and arbitrary function $g\left(s_{r}\right)$ be defined as $\tilde{g}\left(s_{w}\right)$, and the composite function of $s_{u}\left(s_{w}\right)$ 
and arbitrary function $g\left(s_{w}\right)$ be defined as $\hat{g}\left(s_{w}\right)$. From Eq. (2), the object coordinate system of the ridge and upper lines is expressed as

$$
\begin{gathered}
{\left[\begin{array}{lll}
\tilde{\boldsymbol{\xi}}_{R}^{\prime} & \tilde{\boldsymbol{\eta}}_{R}^{\prime} & \tilde{\boldsymbol{\zeta}}_{R}^{\prime}
\end{array}\right]=s_{r}^{\prime}\left[\begin{array}{lll}
\tilde{\boldsymbol{\xi}}_{R} & \tilde{\boldsymbol{\eta}}_{R} & \tilde{\boldsymbol{\zeta}}_{R}
\end{array}\right]\left[\begin{array}{ccc}
0 & -\tilde{\omega}_{\zeta_{R}} & \tilde{\omega}_{\eta_{U}} \\
\tilde{\omega}_{\zeta_{U}} & 0 & -\tilde{\omega}_{\xi_{U}} \\
-\tilde{\omega}_{\eta_{U}} & \tilde{\omega}_{\xi_{U}} & 0
\end{array}\right],} \\
{\left[\begin{array}{lll}
\hat{\boldsymbol{\xi}}_{U}^{\prime} & \hat{\boldsymbol{\eta}}_{U}^{\prime} & \hat{\boldsymbol{\zeta}}_{U}^{\prime}
\end{array}\right]=s_{u}^{\prime}\left[\begin{array}{lll}
\hat{\boldsymbol{\xi}}_{U} & \hat{\boldsymbol{\eta}}_{U} & \hat{\boldsymbol{\zeta}}_{U}
\end{array}\right]\left[\begin{array}{ccc}
0 & -\hat{\omega}_{\zeta_{U}} & \hat{\omega}_{\eta_{U}} \\
\hat{\omega}_{\zeta_{U}} & 0 & -\hat{\omega}_{\xi_{U}} \\
-\hat{\omega}_{\eta_{U}} & \hat{\omega}_{\xi_{U}} & 0
\end{array}\right],}
\end{gathered}
$$

and position $\boldsymbol{x}_{R}\left(s_{r}\right) \equiv \tilde{\boldsymbol{x}}_{R}\left(s_{w}\right)$ and $\boldsymbol{x}_{U}\left(s_{u}\right) \equiv \hat{\boldsymbol{x}}_{U}\left(s_{w}\right)$ are expressed as

$$
\begin{aligned}
& \tilde{\boldsymbol{x}}_{R}\left(s_{w}\right)=\int_{0}^{s_{w}} \tilde{\boldsymbol{\zeta}}_{R} s_{r}^{\prime} d s_{w}, \\
& \hat{\boldsymbol{x}}_{U}\left(s_{w}\right)=\int_{0}^{s_{w}} \hat{\boldsymbol{\zeta}}_{U} s_{u}^{\prime} d s_{w} .
\end{aligned}
$$

From above, the cup shape is determined by functions $\omega_{R}, \omega_{U}, s_{r}$, and $s_{u}$.

The Ritz method and non-Linear programming method are used to solve this optimization. Each function is expressed by the Ritz method (Elsgolc, 1961). For example, $\omega_{\eta_{R}}$ is donated as

$$
\omega_{\eta_{R}}=\sum_{i=0}^{n-1} a_{i} e_{i}=\boldsymbol{a}_{\eta_{R}} \cdot \boldsymbol{e},
$$

by a weighted linear sum of the basis functions. Here, we use trigonometric functions with different cycles. When the coefficient vectors of each function are collectively denoted by $\boldsymbol{a}_{\mathrm{all}}=\left[\boldsymbol{a}_{\omega_{\eta_{R}}}, \cdots, \boldsymbol{a}_{v_{U}}\right]$, the previously formulated optimization problem is a nonlinear programming problem with respect to $\boldsymbol{a}_{\text {all }}$, which is solved using the multiplier and Nelder-Mead methods in this study. Solving the nonlinear programming problem can achieve the objective of this paper.

\section{Simulation and Verification}

\subsection{Results}

This section verifies our proposed method using numerical and experimental results. In Example 1, the data point, whose shape is composed of two developable surfaces, is given. This example aims to confirm that developable surfaces can be reconstructed by our proposed method. For the example, the wire line given as the infinitesimal rotational ratios written as

$$
\omega_{W}=\left[\begin{array}{lll}
0 & 2.91 & 0
\end{array}\right]^{T}
$$

while connection point $\boldsymbol{X}_{C} \in \mathbb{R}^{3}$ is given as

$$
\boldsymbol{X}_{C}=\left[\begin{array}{lll}
0 & 0.34 & 0.34
\end{array}\right]^{\mathrm{T}} \text {. }
$$

Example 2 is a sphere, which is known as non-developable surface. The data points for the surface are given. The example aims to confirm that our proposed method can design the developable surfaces approximating data points. The wire line and connect point was the same as those in Example 1.

In Example 3, the data points from the measured shape are given. When designing a two-piece brassiere cup, it is must fit a breast shape. In this experiment, we measured the breast shape of a torso. The wire line is the same as that in Example 1 and the target connection point is given as

$$
\boldsymbol{X}_{C}=\left[\begin{array}{lll}
0.22 & 0.40 & 0.27
\end{array}\right]^{\mathrm{T}}
$$

In this example, a brassiere cup is assumed to cover only the area of the breast area of in which the y-coordinate is less than 0.2 (Fig.7). In Fig.7, $L_{M}=72.8$ corresponds to the scale factor to fit the wire shape in Example 3 to those in Examples 1 and 2. Example 3 aims to confirm that the propose method can approximate a realistic breast shape. The quantity of data points in each example is 3200,1000 and 1704 .

Figures 8, 10, and 12 show the input data and obtained surfaces in Examples 1, 2, and 3, respectively. In each figure, (a),(b), and (c) show the zx, zy, and xy-views, respectively, while (d) shows the computational surface model, and (e) shows the input data points with colored by the error between the obtained surfaces, the obtained boundary lines, and given connection point. Figures 7, 9, and 11 show developed shapes of patterns for Examples 1, 2, and 3, respectively. 

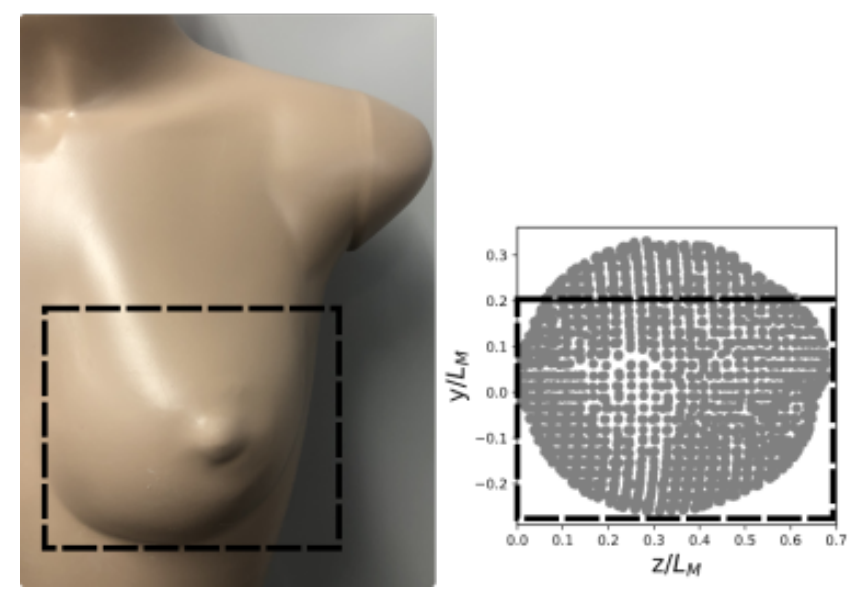

Fig. 7 Border line to use as the point cloud in the experiment.

In each figure, the solid and dotted lines denote the boundary curves of $\partial S_{L}$ and $\partial S_{U}$, respectively. Table 1 shows $\Lambda(\boldsymbol{D})$, the maximum value of the error $\max _{(\boldsymbol{p} \in \boldsymbol{D})} \varepsilon(\boldsymbol{p})$, the average value of the error $\bar{\varepsilon}(\boldsymbol{p})_{\boldsymbol{p} \in \boldsymbol{D}}$ and the calculation time in each example. This problem was implemented by $\mathrm{C}++$ language, the library of vector operation Eigen(G..Guennebaud et al., 2010), and Visual Studio 2019 and and performed on a 3.2GHz, Intel(R) Core(TM) i7-8700 CPU with 16GB Memory operated by Windows 10 Pro.

Table 1 The error and calculation time of our proposed method in each example. Note that the errors of Example 1 and Example 2 are normalized by the length of a wire line and those of Example 3 are normalized by the scale factor $L_{M}$

\begin{tabular}{|c|c|c|c|c|c|}
\hline- & $\Lambda(\boldsymbol{D})$ & $\max _{(\boldsymbol{p} \in \boldsymbol{D})} \varepsilon(\boldsymbol{p})$ & $\bar{\varepsilon}(\boldsymbol{p})_{\boldsymbol{p} \in \boldsymbol{D}}$ & $\left|\boldsymbol{X}_{C}-\boldsymbol{x}_{U}\left(s_{c}\right)\right|^{2}$ & Time $[\mathrm{Sec}]$ \\
\hline Example 1 & $1.12 \times 10^{-1}$ & $2.41 \times 10^{-4}$ & $3.49 \times 10^{-5}$ & $8.26 \times 10^{-4}$ & $1.8 \times 10^{3}$ \\
\hline Example 2 & $5.99 \times 10^{-2}$ & $6.95 \times 10^{-4}$ & $5.99 \times 10-5$ & $5.52 \times 10^{-5}$ & $7.40 \times 10^{2}$ \\
\hline Example 3 & $7.57 \times 10^{-1}$ & $1.25 \times 10^{-2}$ & $4.44 \times 10^{-4}$ & $3.94 \times 10^{-3}$ & $1.68 \times 10^{3}$ \\
\hline
\end{tabular}

\subsection{Discussion}

Figure 8, demonstrates that our method can reconstruct two developable surfaces from a data cloud. In particular, the ridge line is reconstructed precisely. In the design of a structure consisting of multiple developable surfaces, the boundaries of the individual surfaces must be set properly. Our proposed method can optimize the position and shape of such boundaries.

Figures 10 and 11 confirm the effectiveness of our method. The spherical surface can be approximately formed by connecting several fusiform-shaped patterns like a terrestrial globe. The almost fusiform-shaped patterns are derived automatically.

Example 3 has a larger error and requires a longer calculation time than Example 2. This is attributed to the difference in shape; a realistic breast has a more complex shape than a sphere. To fit only two developable surfaces to such a complex shape, patterns with complex shapes were obtained (Fig.13). Furthermore, the upper line is optimized to prioritize minimizing the objective function over passing close by the target connecting point. Note that the proposed method does not guarantee that a derived boundary curve is smooth. So, there can be a tangential discontinuity point on the dotted upper edge as shown in Fig.13b due to the combination of the obtained length of a generatrix, its angle, and geodesic curvature of the lower edge. To get a smooth boundary curve, only the obtained lengths of the generatrices needs to be fine-tuned while it leads to slight change of the size of covered area with a developable surface.

Hence, our proposed method is useful to efficiently design developable surfaces. 
Yoshida, Wakamatsu, Morinaga, Tsutsumi and Kubo, Journal of Advanced Mechanical Design, Systems, and Manufacturing, Vol.15, No.5 (2021)

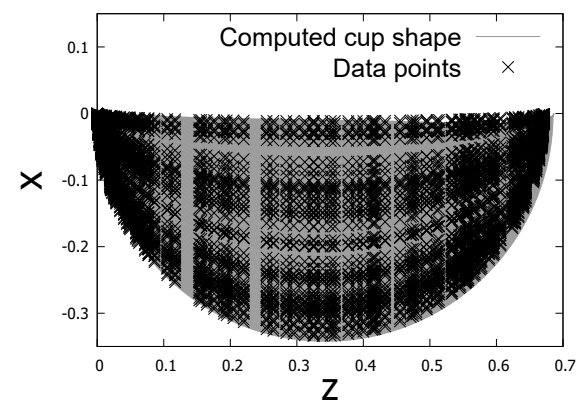

(a) zx-View

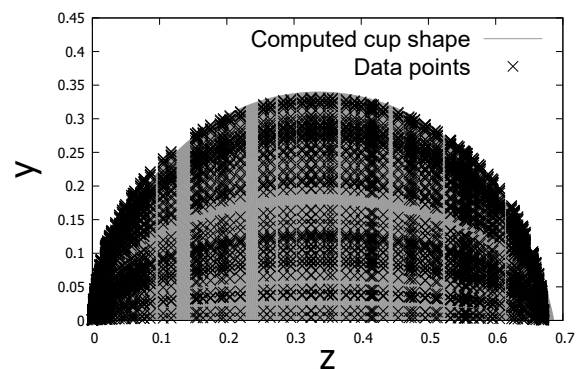

(b) zy-View

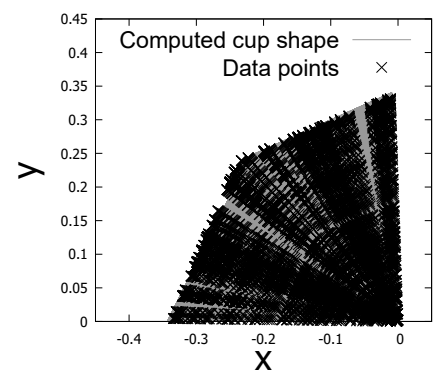

(c) $x y-V i e w$

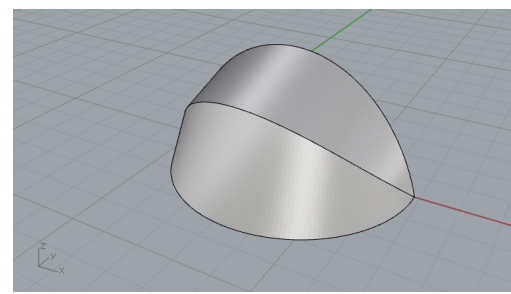

(d) Computational surface model in perspective view

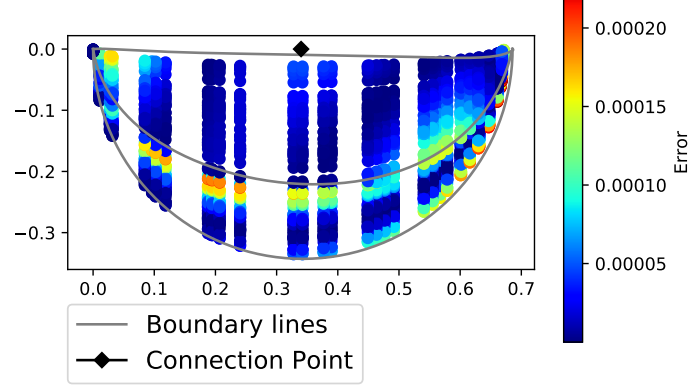

(e) Input data points with colored by the error

Fig. 8 Obtained result of Example 1 by our proposed method. Input data points are the shape of the two developable surfaces. (a),(b),(c) Calculated shape and given data points in each view, and (d) its computational surface model in the perspective view. Note that this surface is shaded to make it look smooth. (e) the input data points with colored by the error between the obtained surfaces.

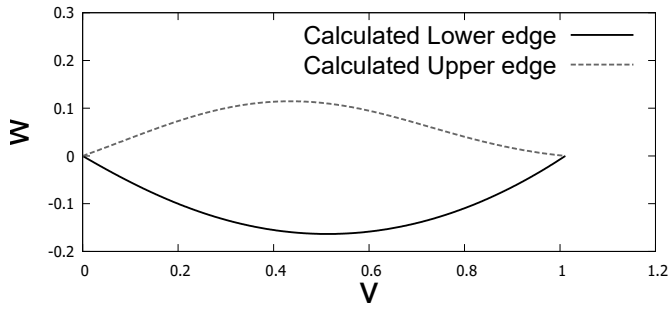

(a) Lower pattern

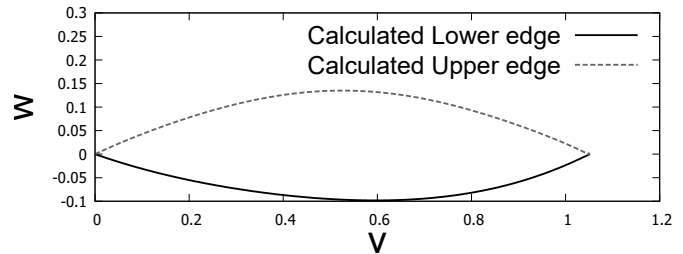

(b) Upper pattern

Fig. 9 Obtained developed shape from the result of Example 1 where (a) and (b) correspond to the lower and upper pattern of a two-piece brassiere cup, respectively. 
Yoshida, Wakamatsu, Morinaga, Tsutsumi and Kubo, Journal of Advanced Mechanical Design, Systems, and Manufacturing, Vol.15, No.5 (2021)

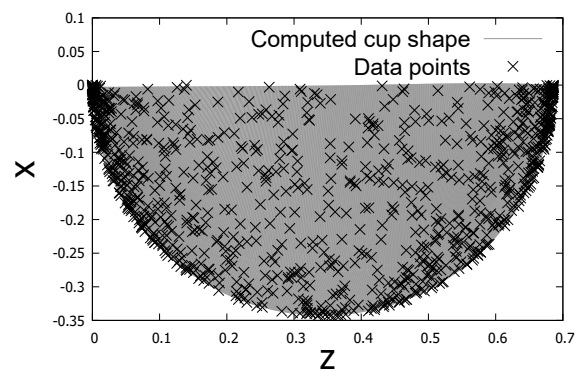

(a) zx-View

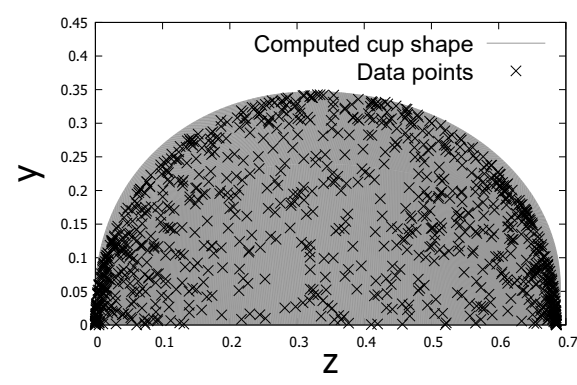

(b) zy-View

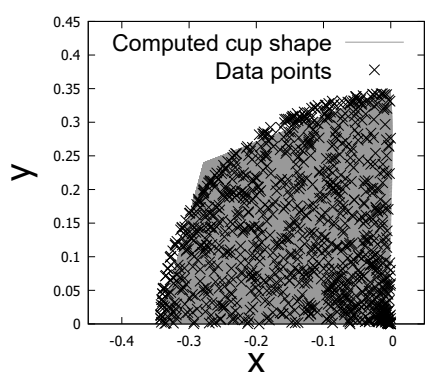

(c) xy-View

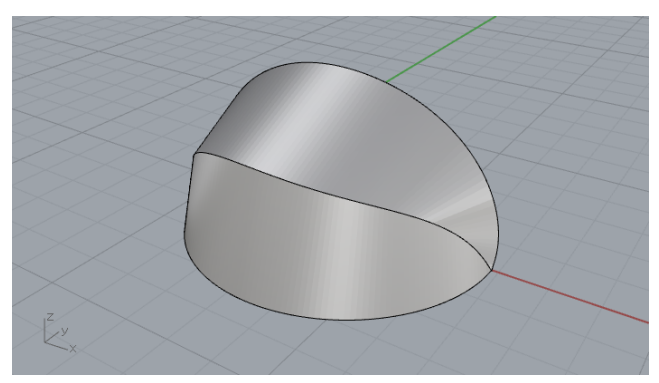

(d) Computational surface model in perspective view

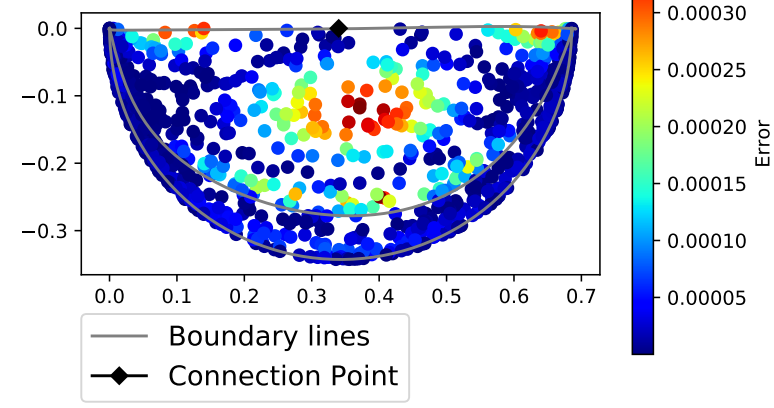

(e) Input data points with colored by the error

Fig. 10 Obtained result of Example 2 by our proposed method. Input data points are the shape of the two developable surfaces. (a),(b),(c) Calculated shape and given data points in each view, and (d) its computational surface model in the perspective view. Note that this surface is shaded to make it look smooth. (e) the input data points with colored by the error between the obtained surfaces.

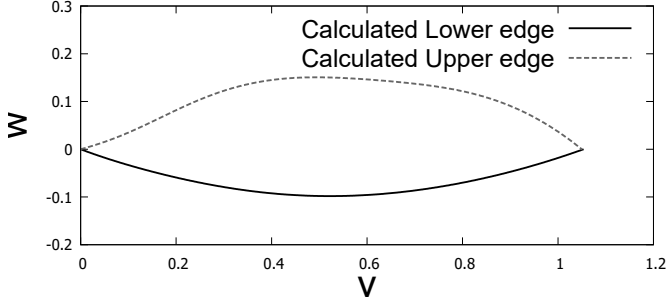

(a) Lower pattern

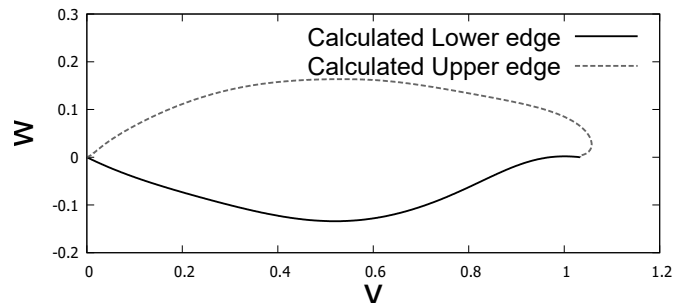

(b) Upper pattern

Fig. 11 Obtained developed shape from the result of Example 2 where (a) and (b) correspond to the lower and upper pattern of a two-piece brassiere cup, respectively. 
Yoshida, Wakamatsu, Morinaga, Tsutsumi and Kubo, Journal of Advanced Mechanical Design, Systems, and Manufacturing, Vol.15, No.5 (2021)

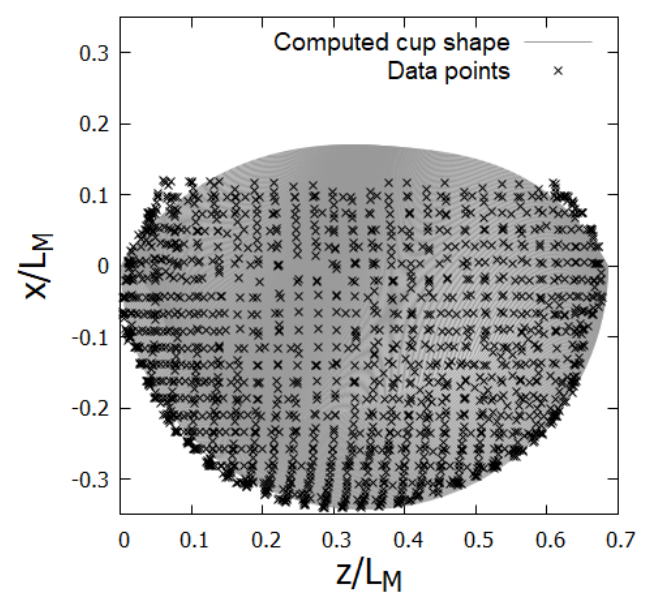

(a) zx-View

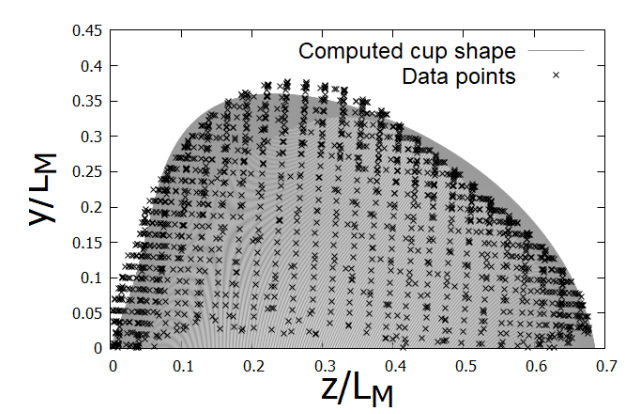

(b) zy-View

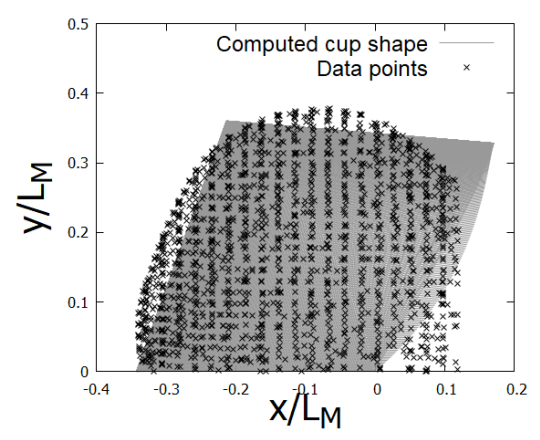

(c) $x y-V i e w$

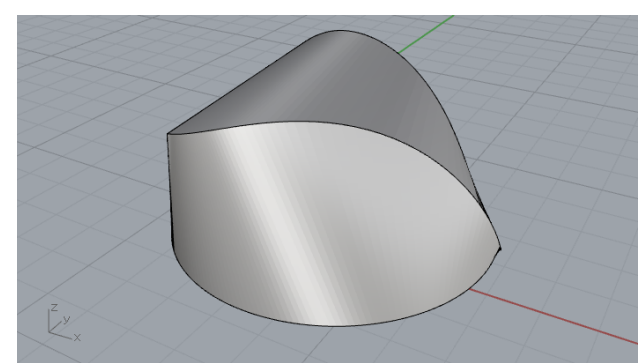

(d) Computational surface model in perspective view

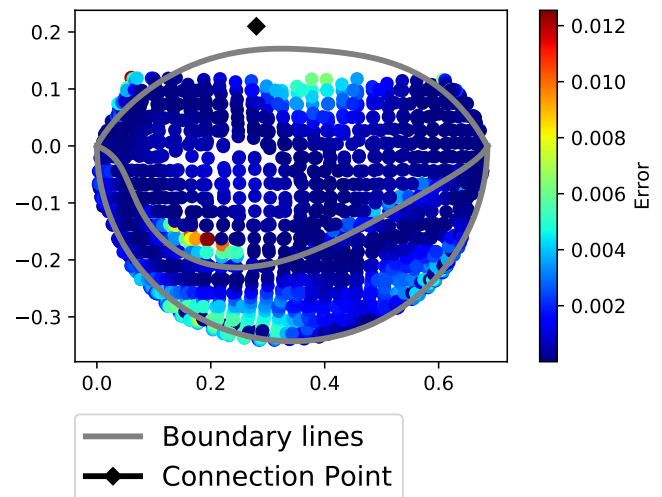

(e) Input data points with colored by the error

Fig. 12 Obtained result of Example 3 by our proposed method. Input data points are the shape measured by the breast shape of a torso as shown in Fig.7. (a),(b),(c) Calculated shape and given data points in each view, and (d) its computational surface model in the perspective view. Note that this surface is shaded to make it look smooth. (e) the input data points with colored by the error between the obtained surfaces. 


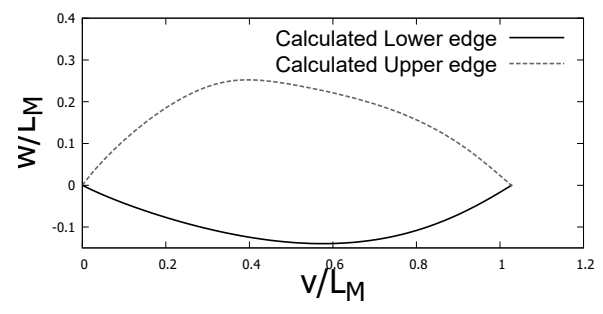

(a) Lower pattern

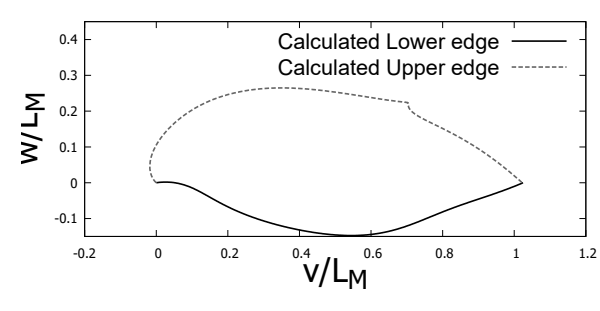

(b) Upper pattern

Fig. 13 Obtained developed shape from the result of Example 3 where (a) and (b) correspond to the lower and upper pattern of a two-piece brassiere cup, respectively.

\subsection{Limitation and future work}

In this study, we propose a method to design developable surfaces from the intended data points and verified its validity by applying it to the design process of a two-piece brassiere cup as a case study. Here we discuss the limitation and future work of this study.

The proposed method is applicable to approximation of a curved surface from its data points with more than three developable surfaces, which are enclosed by two main boundary curves and connected at those curves in tandem as shown in Fig.14-(a), which each surface can be determined by two boundary curves. The shape of boundary curves can be determined simultaneously with the proposed method. However, the method cannot be applied to approximation with developable surfaces, some of which are enclosed by more than three main boundary curves and connected not in tandem as shown in Fig.14-(b). Wakamatsu etc. proposed a method to calculate a three-dimensional shape from its pattern shapes for the efficiency of design process of a three-piece brassiere cup(Wakamatsu et al., 2017). According to it, when calculating a 3D surface shape from a pattern shape, a triangular planar area may appear in the center of the deformed pattern if the pattern has three main boundary curves as shown in Fig.14-(b). In this case, some generatrices are spanned between boundary 1 and boundary 2, but the others are spanned between boundary 1 and boundary 3 , for example. This means that the whole shapes of boundary 2 and boundary 3 cannot be determined completely from the shape of boundary 1 even if the proposed method is applied. Thus, some extension of the proposed method is needed if you want to approximate a curved surface with meshes of developable surfaces as shown in Fig.14-(b). Since the objective function of this method is the summation of the error, its calculation time and value are proportional to the quantity of the data points $N$. If $N$ is too large, computational cost is increased. But when making $N$ smaller, the problem to determine how data points are distributed in Euclidian space appears. When the data points do not express the feature of the intended shape well, it may lead to unexpected results. This problem is a subject for the future analysis.

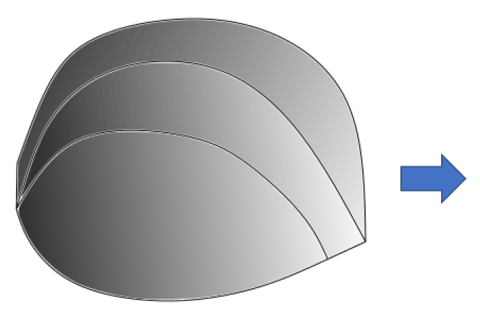

(a) our proposed method can deal with

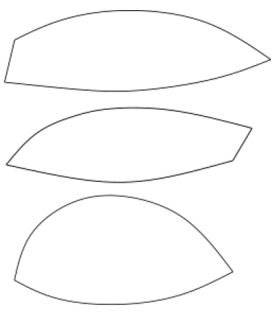

Fig. 14 The extended examples of our proposed method One can be dealt with our proposed method. The other cannot be dealt with it.

\section{Conclusion}

Our proposed method efficiently designed the shape of developable surfaces when the data points are given. First, we formulated a developable surface based on the differential geometry. Second, we hypothesized that two boundary curves could determine a developable surface as an optimization problem where the objective function is the error between the 
developable surface and the data points. Third, we calculated the error between a point and the surface between the geometry condition. Our proposed method divided the data cloud into two for a two-piece brassiere cup: the lower and upper cups. Then the shape of the boundary curve and the two developable surfaces were optimized. Our proposed method was verified using three examples. The data points in Example 1 corresponded to two developable surfaces. They corresponded to the surface of a sphere in Example 2, while those in Example 3 corresponded to the surface of a realistic breast. In all cases, the developable surfaces were optimized. and their developed shapes fit the given data points, demonstrating that our method can efficiently design a developable surface.

\section{References}

Aumann, G., A simple algorithm for designing developable Bezier surfaces, in Computer Aided Geometrix Design Volume 20(2003), pp.601-619.

Bo, P. and Wang, W., Geodesic-Controlled Developable Surfaces for Modeling Paper Bending, in Computer Graphics Forum, Volume 26(2007), pp.365-374.

Bodduluri, R.M.C and Ravani, B., Design of developable surfaces using duality between plane and point geometries, in Computer Aided Design Volume 25(1993), pp.621-632.

Chen, H.-Y., Lee, I.-K., Leopoldseder, S., Pottmann, H., Randrup, T., Wallner, J., On Surface Approximation using Developable Surfaces, Graphical Models and Image Processing, Volume 61, Issue2(1999), pp.110-124

Elsgolc L.E, Calculus of Variations, Pergamon Press(1961), pp. 144-157.

Guennebaud, G., Jacob, B. and others, Eigen v3, http://eigen.tuxfamily.org, 2010

Harashima, A., "Rikigaku", Shokabo Co., Ltd.(1985), pp. 207 (Japanese).

Itoh, M., and Imaoka, H., A Method of Prediction Sewn Shapes and a Possibility of Sewing by the Theory of Developable Surfaces, J. the Japan Research Association for Textile End-Uses, Volume 48(2007), pp.42-51, (Japanese).

Kilian, M., Flöry, S., Chen, Z., Mitra, N. J., Sheffer, A., and Pottmann, H., Curved folding, ACM Transaction on Graphics, Volume27, Issue3(2008), pp1-9

Kobayashi, S., Differential Geometry of Curves and Surface, Shokado, Tokyo(1995), Chap2 (in Japanse)

Liu, Y., Pottmann, H., Wallner, J., Yang, Y.-L., and Wang, W., Geometric modeling with conical meshes and developable surfaces, Proc.Int. Symp. SIGGRAPH(2006), pp.681-689

Mitani, J., A Design Method for 3D Origami Based on Rotational Sweep, Computer-Aided Design and Applications, vol.6(1)(2009), pp.69-79

Peternell, M., Developable surface fitting to point clouds, in Computer Aided Design Vol.21(2004), pp.785-803.

Pottmann, H. and Wallner, J., Approximation algorithms for developable surfaces, in Computer Aided Geometric Design, Volume 16(1999), pp.539-556.

Ravani, B. and Ku, T.S., Bertrand offsets of ruled and developable surfaces in Computer-Aided Design Volume 23(1991), pp.145-152.

Rose, K., Sheffer, A., Wither, J., Cani, M., and Thibert, B., Developable surfaces from arbitrary sketched boundaries, SGP '07 Proceedings of the fifth Eurographics symposium on Geometry processing(2007), pp.163-172.

Stein, O., Grinspun, E., and Crane, K., Developability of triangle meshes, ACM Transaction on Graphics, Volume 37, Issue4(2018), pp77:1-14

Wakamatsu, H., Ryu, Y., Morinaga, E., Arai, E., and Kubo, T., Oyama, M., and Kishimoto, T., 3D Shape Prediction of A Paper Model of Two Piece Brassiere Cup for Its Efficient Design, Proc.Int. Symp. Flexible Automation(2016), pp.326-332

Wakamatsu, H., Morinaga, E., Arai, E., and Kubo, T., A Virtual Paper Model of a Three Piece Brassiere Cup to Improve the Efficiency of Cup Design Process, Proc. 2017 IEEE Int. Conf. on Robotics and Automation(2017), pp. 11691174.

Yoshida, K., Wakamatsu, H., Morinaga, E., Arai, E., Tsutsumi, S., Kubo, T., Pattern Shape Optimization of a Two-piece brassiere cup to improve its design efficiency, Trans. of the Institute of Systems, Control and Information Engineers, Volume 32, No. 5(2019), pp. 192-202.

Yoshida, K., Wakamatsu, H., Morinaga, E., Tsutsumi, S., and Kubo, T., Design support of a developable surface considering its bending energy, Transaction of the JSME, Vol.86, No.890(2020), DOI: 10.1299/transjsme.20-00169 (in Japanese) 